\title{
Large amplitude electromagnetic solitons in a fully relativistic magnetized electron-positron-pair plasma
}

\author{
Gadadhar Banerjee ${ }^{\mathrm{a}, *}$, Sayantan Dutta ${ }^{\mathrm{b}, \mathrm{c}}$, A. P. Misra ${ }^{\mathrm{a}, * *}$ \\ ${ }^{a}$ Department of Mathematics, Siksha Bhavana, Visva-Bharati (A Central University), Santiniketan-731 235, India \\ ${ }^{b}$ Laboratoire de Physique Thorique, IRSAMC, UMR CNRS 5152, Universit Paul Sabatier, 31062 Toulouse, France \\ ${ }^{c}$ Institut de Recherche en Informatique de Toulouse, UMR CNRS 5505, Universit de Toulouse, 31062 Toulouse, France
}

\begin{abstract}
Nonlinear propagation of purely stationary large amplitude electromagnetic (EM) solitary waves in a magnetized electron-positron (EP) plasma is studied using a fully relativistic two-fluid hydrodynamic model which accounts for physical regimes of both weakly relativistic $\left(P \ll n m c^{2}\right)$ and ultrarelativistic $\left(P \gg n m c^{2}\right)$ random thermal energies. Here, $P$ is the thermal pressure, $n$ the number density and $m$ the mass of a particle, and $c$ is the speed of light in vacuum. While both the sub-Alfvénic and super-Alfvénic solitons coexist in the weakly relativistic regime, the ultrarelativistic EP plasmas in contrast support only the subAlfvénic solitons. Different limits of the Mach numbers and soliton amplitudes are also examined in these two physical regimes.
\end{abstract}

Keywords: Electron-positron plasma, Relativistic plasma, Alfvénic soliton, pseudopotential

\section{Introduction}

Electron-positron (EP) plasmas have been known to play important roles in many physical situations, such as active galactic nuclei (Begelman et al., 1984; Miler and Witta, 1987), pulsars (Goldreich and Julian, 1969), quasars (Wardle et al., 1998), black holes (Blandford and Znajek. 1977), accretion disks (Orosz et al., 1997), the early universe (Misner et al., 1973: Gibbons et al., 1983), near the polar cap of fast rotating neutron stars (Lightman, 1982, Burns and Lovelace, 1982, Lightman and Zdziarski, 1987; Yu et al., 1986), as well as in laboratories (Sarri et al. 2015). In the latter, it has been shown that the production of the ionfree high-density neutral EP-pair plasmas and their identification as collective modes can be possible in a controlled laboratory environment.

\footnotetext{
* On leave from Department of Basic Science and $\mathrm{Hu}-$ manities, University of Engineering \& Management (UEM), Kolkata-700 160.

** Corresponding author

Email addresses: gban.iitkgp@gmail.com (Gadadhar

Banerjee), dutta@irsamc.ups-tlse.fr;

sayantan.dutta110@gmail.com (Sayantan Dutta),

apmisra@visva-bharati.ac.in; apmisra@gmail.com (A. P. Misra)
}

Preprint submitted to Elsevier
Linear and nonlinear waves in EP-pair plasmas differ fundamentally from those in ordinary electron-ion plasmas or from a purely electronic beam due to their intrinsic and complete symmetry with equal charge (but opposite in sign) and mass. The Sagdeev or pseudopotential approach has been the most suitable technique for the desription of nonlinear large amplitude waves (Misra and Adhikary, 2011, 2013, Sagdeev, 1966; Sagdeev and Galeev, 1969 Baboolal et al., 1990 Mace et al. 1991; Banerjee and Maitra, 2015, 2016; Saini et al. 2011; Das et al., 2010) which also works well in pair plasmas (Verheest et al., 1996). However, when relativistic dynamics is included together with thermal pressure of plasma particles for the description of large amplitude EM waves, the Sagdeev's approach may not be suitable. In this context, an alternative procedure has also been developed by McKenzie et al. (McKenzie and Doyle, 2003) to study the properties of nonlinear waves in its own frame of reference. Although both approaches are analogous to each other especially for electrostatic waves, the McKenzie approach provides a better perception and usefulness than the Sagdeev's approach especially when one is concerned with the propagation of electromagnetic (EM) solitary waves in plasmas 
(Verheest and Cattaert, 2004, 2005). In the latter, Verheest and Cattaert have studied the propagation of large amplitude EM waves in nonrelativistic and relativistic EP-pair plasmas without any thermal flow of electrons and positrons using the McKenzie approach.

In this work, our aim is to advance and generalize the theory of Verheest and Cattaert (Verheest and Cattaert, 2004) by considering the fully relativistic fluid models for electrons and positrons which account for physical regimes of both weakly relativistic and ultrarelativistic random thermal energies. We show that in contrast to the weakly realtivistic plasmas which support both sub-Alfvénic and super-Alfvénic solitons, only the sub-Alfvénic solitons can be formed in EP-pair plasmas with ultrarelativistic energies.

\section{Relativistic fluid model and multispecies integrals}

We consider the nonlinear propagation of EM solitary waves along the constant magnetic field $B_{0} \hat{x}$ in an EP-pair plasma with relativistic flow of thermal electrons and positrons. We assume that the effective collision frequency in an EP-pair plasma, which includes the recombination and photon annihilation effects, is assumed to be much smaller than the plasma oscillation frequency of electrons and positrons. From the energy momentum tensor, the basic equations for the relativistic dynamics of a $j$-th species particle can be written as (Gratton et al. 1997; Gomberoff and Galvão, 1997)

$$
\begin{aligned}
& \frac{\partial}{\partial t}\left(\gamma_{j} n_{j}\right)+\nabla \cdot\left(\gamma_{j} n_{j} \mathbf{v}_{j}\right)=0, \\
& \frac{H_{j}}{c^{2}}\left(\frac{\partial}{\partial t}+\mathbf{v}_{j} \cdot \nabla\right)\left(\gamma_{j} \mathbf{v}_{j}\right)=n_{j} q_{j}\left(\mathbf{E}+\frac{1}{c} \mathbf{v}_{j} \times \mathbf{B}\right) \\
& -\frac{1}{\gamma_{j}} \nabla P_{j}-\frac{\mathbf{v}_{j} \gamma_{j}}{c^{2}} \frac{d P_{j}}{d t} \\
& \frac{1}{n_{j}} \frac{d P_{j}}{d t}=\frac{d}{d t}\left(\frac{H_{j}}{n_{j}}\right),
\end{aligned}
$$

where $d / d t \equiv \partial_{t}+\mathbf{v}_{j} \cdot \nabla, n_{j}, q_{j}, m_{j}, \mathbf{v}_{j}, \gamma_{j}$, $P_{j}$ and $H_{j}$ are, respectively, the number density, charge, mass, fluid velocity, relativistic factor, thermal pressure and enthalpy per unit volume of $j$ species particle. Also, $\mathbf{E}$ and $\mathbf{B}$ are the electric and magnetic (total) fields respectively. Introducing $\mathcal{E}_{j}$ as the total energy density and $\epsilon_{j}$ the internal energy density of the $j$-species fluid, we have
$H_{j}=\mathcal{E}_{j}+P_{j}$ and $\mathcal{E}_{j}=n_{j} m_{j} c^{2}+\epsilon_{j}$. We consider the ploytropic pressure law as (Gratton et al., 1997. Gomberoff and Galvão, 1997) $P_{j}=(\Gamma-1) \epsilon_{j}=$ $n_{j} k_{B} T_{j}$, where $k_{B}$ is the Boltzmann constant, so that $\epsilon_{j}=n_{j} k_{B} T_{j} /(\Gamma-1)$ and $H_{j} \equiv n_{j} \alpha_{j}=$ $n_{j} m_{j} c^{2}+\Gamma P_{j} /(\Gamma-1)=n_{j} m_{j} c^{2}\left[1+\Gamma \beta_{j} /(\Gamma-1)\right]$ with the energy ratio $\beta_{j}=k_{B} T_{j} / m_{j} c^{2}$ and the polytropic index $4 / 3 \leq \Gamma \leq 5 / 3$. In particular, $\Gamma=5 / 3$ and $4 / 3$, respectively, correspond to the weakly relativistic (classical) and ultrarelativistic regimes. So, in the weakly relativistic limit $P_{j} \ll n_{j} m_{j} c^{2}$ (applicable for low-energy plasmas) we have for $\Gamma=5 / 3, H_{j}=n_{j} m_{j} c^{2}+(5 / 2) n_{j} k_{B} T_{j} \approx$ $n_{j} m_{j} c^{2}$, and in the regime of ultrarelativistic energies where $P_{j} \gg n_{j} m_{j} c^{2}$, we have instead $H_{j}=$ $n_{j} m_{j} c^{2}+4 n_{j} k_{B} T_{j} \approx 4 n_{j} k_{B} T_{j}$.

The system is then closed by the following Maxwells equations.

$$
\begin{gathered}
\nabla \cdot \mathbf{E}=4 \pi \sum_{j} q_{j} n_{j} \gamma_{j}, \\
\nabla \cdot \mathbf{B}=0 \\
\nabla \times \mathbf{E}=-\frac{1}{c} \frac{\partial \mathbf{B}}{\partial t}, \\
\nabla \times \mathbf{B}=\frac{4 \pi}{c} \sum_{j} q_{j} n_{j} \gamma_{j} \mathbf{v}_{j}+\frac{1}{c} \frac{\partial \mathbf{E}}{\partial t} .
\end{gathered}
$$

In order to derive an evolution equation for purely stationary nonlinear solitary EM waves and their properties from Eqs. (1) to (7) we follow the McKenzie approach as used in, e.g., Ref. (Verheest and Cattaert, 2004, 2005). First, we derive various conserved quantities for a general species $j$ before we apply it for an EP plasma. We look for the excitation of solitary waves that propagate along the constant magnetic field $\mathbf{B}_{0}$, i.e., the $x$-axis. In a frame moving with the constant speed $V$ along the $x$-axis, all plasma species have the same constant velocity $V$ along the direction. Since in the wave frame there is no time derivative, Eqs. (1) and (2) reduce to

$$
\begin{gathered}
\frac{d}{d x}\left(\gamma_{j} n_{j} \mathbf{v}_{j x}\right)=0 \\
\frac{\alpha_{j}}{c^{2}} \gamma_{j} n_{j} v_{j x} \frac{d}{d x}\left(\gamma_{j} \mathbf{v}_{j}\right)=\gamma_{j} n_{j} q_{j}\left(\mathbf{E}+\frac{1}{c} \mathbf{v}_{j} \times \mathbf{B}\right) \\
-\frac{d P_{j}}{d x} \hat{x} .
\end{gathered}
$$


Also, from Eqs. (4) to (7) we successively obtain the following equations.

$$
\begin{gathered}
\frac{d E_{x}}{d x}=4 \pi \sum_{j} q_{j} n_{j} \gamma_{j}, \\
\frac{d B_{x}}{d x}=0, \\
\hat{x} \times \frac{d \mathbf{E}}{d x}=0, \\
\hat{x} \times \frac{d \mathbf{B}}{d x}=\frac{4 \pi}{c} \sum_{j} q_{j} n_{j} \gamma_{j} \mathbf{v}_{j}
\end{gathered}
$$

Now, Eq. 11 gives on integration $B_{x}=B_{0}$, a constant. Also, from Eq. 12 it follows that $\mathbf{E}_{\perp}=$ 0 under the boundary condition $\mathbf{E} \rightarrow 0$ as $x \rightarrow \pm \infty$, and so only $E_{x}=d \phi / d x$ ( $\phi$ is the scalar potential) and $\mathbf{B}_{\perp}$ are the variables, which also tend to zero as $x \rightarrow \pm \infty$, i.e., in the undisturbed plasma far away from the region of the nonlinear structure. Next, from the equation of continuity (8), we obtain the following conservation of mass (parallel flux).

$$
\gamma_{j} n_{j} v_{j x}=\gamma_{j 0} n_{j 0} V
$$

From Eq. (9), after summing over all the species and using Eqs. (10), (13) and $(14)$, we obtain

$$
\begin{aligned}
\frac{V}{c^{2}} \sum_{j} \gamma_{j 0} n_{j 0} \alpha_{j} \frac{d}{d x}\left(\gamma_{j} \mathbf{v}_{j}\right) & =\frac{1}{4 \pi}\left[E_{x} \frac{d E_{x}}{d x}+\left(\hat{x} \times \frac{d \mathbf{B}_{\perp}}{d x}\right)\right. \\
& \times \mathbf{B}]-\sum_{j} \frac{d P_{j}}{d x} \hat{x} .
\end{aligned}
$$

Integrating Eq. 15 with respect to $x$ we obtain the following two distinct integrals of motion.

$$
\begin{aligned}
\frac{V}{c^{2}} \sum_{j} \gamma_{j 0} n_{j 0} \alpha_{j}\left(\gamma_{j} v_{j x}-\gamma_{j 0} V\right) & =\frac{1}{8 \pi}\left(E_{x}^{2}-B_{\perp}^{2}\right) \\
& -\sum_{j}\left(P_{j}-P_{j 0}\right) \\
\frac{V}{c^{2}} \sum_{j} \gamma_{j 0} n_{j 0} \alpha_{j} \gamma_{j} \mathbf{v}_{j \perp} & =\frac{B_{0}}{4 \pi} \mathbf{B}_{\perp}
\end{aligned}
$$

Furthermore, the projection of Eq. 9 on $\mathbf{v}_{j \perp}$ gives $\mathbf{v}_{j \perp} \cdot\left[\frac{\gamma_{j} n_{j} \alpha_{j}}{c^{2}} \frac{d}{d x}\left(\gamma_{j} \mathbf{v}_{j \perp}\right)\right]=\frac{q_{j} \gamma_{j} n_{j}}{c} \mathbf{v}_{j \perp} \cdot\left(\mathbf{e}_{x} \times \mathbf{B}_{\perp}\right)$
Multiplying Eq. (18) by $\gamma_{j 0} \alpha_{j} n_{j 0} / q_{j}$, summing over all the species and integrating we obtain

$$
\sum_{j} \frac{\gamma_{j}^{2} \alpha_{j}^{2} \gamma_{j 0} n_{j 0}}{q_{j}} v_{j \perp}^{2}=0
$$

where we have used Eq. (17). We can also project Eq. (9) on $\mathbf{v}_{j}$ to yield

$$
\frac{\alpha_{j}}{2 c^{2}} \frac{d}{d x}\left(\gamma_{j}^{2} v_{j}^{2}\right)=q_{j} \gamma_{j} E_{x}-K_{B} T_{j} \frac{d}{d x}\left[\log \left(n_{j}\right)\right] .
$$

\section{Relativistic EP plasmas: Energy integral}

We focus our attention to an EP-pair plasma. The results obtained in Sec. 2 will be modified with $q_{e}=-e, q_{p}=e, m_{e}=m_{p}=m, n_{e 0}=n_{p 0}=n_{0}$, $T_{e}=T_{p}=T, \gamma_{e 0}=\gamma_{p 0}=\gamma_{0}$ and $\alpha_{e}=\alpha_{p}=$ $\alpha, \beta_{e}=\beta_{p}=\beta$, where the subscripts $j=e$ and $p$, respectively, stand for electrons and positrons. Thus, for EP plasmas the invariants (14), (16), (17) and 190 , respectively, reduce to

$$
\gamma_{e} n_{e} v_{e x}=\gamma_{p} n_{p} v_{p x}=V \gamma_{0} n_{0},
$$

$\frac{V}{c^{2}} \gamma_{0} n_{0} \alpha\left(\gamma_{e} v_{e x}+\gamma_{p} v_{p x}-2 \gamma_{0} V\right)=\frac{1}{8 \pi}\left(E_{x}^{2}-B_{\perp}^{2}\right)$

$$
-\left(P_{e}+P_{p}-2 P_{0}\right) \text {, }
$$

$$
\begin{gathered}
\frac{V}{c^{2}} \gamma_{0} n_{0} \alpha\left(\gamma_{e} \mathbf{v}_{e \perp}+\gamma_{p} \mathbf{v}_{p \perp}\right)=\frac{B_{0}}{4 \pi} \mathbf{B}_{\perp}, \\
\gamma_{e}^{2} v_{e \perp}^{2}=\gamma_{p}^{2} v_{p \perp}^{2} .
\end{gathered}
$$

Using Eq. 24, we obtain from Eq. 23 the following two results

$$
\begin{gathered}
\left(\gamma_{p} \mathbf{v}_{p \perp}-\gamma_{e} \mathbf{v}_{e \perp}\right) \cdot \mathbf{B}_{\perp}=0 \\
\left(\gamma_{p} \mathbf{v}_{p \perp}+\gamma_{e} \mathbf{v}_{e \perp}\right) \times \mathbf{B}_{\perp}=0 .
\end{gathered}
$$

Thus, it follows from Eqs. 25) and (26) that while the component of $\left(\gamma_{p} \mathbf{v}_{p \perp}-\gamma_{e} \mathbf{v}_{e \perp}\right)$ is orthogonal to $\mathbf{B}_{\perp}$, the other component of $\left(\gamma_{p} \mathbf{v}_{i \perp}+\gamma_{e} \mathbf{v}_{e \perp}\right)$ is parallel to $\mathbf{B}_{\perp}$.

In the weakly nonlinear theory, the truly stationary solutions are only possible at linear polarization of EM fields. So, we can assume without loss of generality that $\mathbf{B}_{\perp}$ is along the $y$-axis. Then Eqs. 25 and 26 give $\gamma_{e} v_{e y}=\gamma_{p} v_{p y}$ and $\gamma_{p} v_{p z}=-\gamma_{e} v_{e z}$, and so the $y$-component of Eq. 13p gives

$$
\sum_{j=e, p} q_{j} n_{j} \gamma_{j} v_{j y}=0
$$


from which one obtains $n_{e}=n_{p}=n$, say. So, form Eq. 21) we have $\gamma_{e} v_{e x}=\gamma_{p} v_{p x}$, and using the charge neutrality condition, $\gamma_{e} n_{e}=\gamma_{p} n_{p}$ we have $\gamma_{e}=\gamma_{p}=\gamma$, say. Thus, we have $v_{e x}=v_{p x}=v_{x}$, $v_{e y}=v_{p y}=v_{y}, v_{e z}=-v_{p z}=v_{z}$ and $v_{p}^{2}=v_{e}^{2}=v^{2}$. Using the charge neutrality condition, the Ampére law 13 reduces to

$$
\mathbf{e}_{x} \times \frac{d \mathbf{B}_{\perp}}{d x}=\frac{4 \pi e n \gamma}{c}\left(\mathbf{v}_{i \perp}-\mathbf{v}_{e \perp}\right)
$$

where $\gamma=1 / \sqrt{1-v^{2} / c^{2}}$. Furthermore, a scalar multiplication of $d \mathbf{B}_{\perp} / d x$ with $\mathbf{B}_{\perp}$ gives

$$
\mathbf{B}_{\perp} \times \frac{d \mathbf{B}_{\perp}}{d x}=0,
$$

meaning that the wave magnetic field $\mathbf{B}_{\perp}$ is linearly polarized. Thus, our assumption of linear polarization of EM fields and quasineutrality condition are valid. Next, from Eqs. 222 and (23), we obtain

$$
\begin{aligned}
\gamma v_{x} & =\frac{1}{2}\left[\gamma_{0} V-\frac{m c^{2} V_{A}^{2}}{2 V \gamma_{0} \alpha} b^{2}+\frac{c^{2} K_{B} T}{V \gamma_{0} \alpha}\right. \\
& \left.+\sqrt{\left(\gamma_{0} V-\frac{m c^{2} V_{A}^{2}}{2 V \gamma_{0} \alpha} b^{2}+\frac{c^{2} K_{B} T}{V \gamma_{0} \alpha}\right)^{2}-\frac{4 c^{2} K_{B} T}{\alpha}}\right]
\end{aligned}
$$

$$
\gamma v_{y}=\frac{m c^{2} V_{A}^{2}}{V \gamma_{0} \alpha} b
$$

where $b=B_{y} / B_{0}$ is the dimensionless wave magnetic field, $\gamma_{0}=1 / \sqrt{1-V^{2} / c^{2}}$ and $V_{A}$ is the Alfvén velocity in an EP plasma, defined by, $V_{A}^{2}=$ $B_{0}^{2} /\left(8 \pi n_{0} m\right)$. Next, rearranging the $y$-component of Eq. (9), we obtain another velocity component

$$
v_{z}=\frac{\alpha v_{x}}{c e B_{0}} \frac{d}{d x}\left(\gamma v_{y}\right)
$$

Note that Eq. 21] results into $n=\left(V \gamma_{0} n_{0}\right) /\left(\gamma v_{x}\right)$ which when applied to Eq. 20 gives, after integration and summation over electron and positron species, the following conservation of kinetic energy.

$$
\gamma^{2}\left(v_{x}^{2}+v_{y}^{2}+v_{z}^{2}\right)=\gamma_{0}^{2} V^{2}-\frac{2 c^{2} K_{B} T}{\alpha} \log \left(\frac{\gamma_{0} V}{\gamma v_{x}}\right)
$$

We define the Mach number as $M=V / V_{A}$ and a dimensionless coordinate $\zeta=x \omega_{p} / c$, where $\omega_{p}^{2} \equiv$ $\omega_{p e}^{2}+\omega_{p p}^{2}=8 \pi n_{0} e^{2} / m$ is the squared total plasma oscillation frequency of electrons and positrons. Finally, using Eqs. 30 to 32 , we obtain from Eq.
(33) the following equation.

$$
\frac{1}{2}\left(\frac{d b}{d \zeta}\right)^{2}+\psi(b)=0
$$

where $\psi$ is the Sagdeev potential or pseudopotential, given by,

$$
\psi(b)=\frac{M^{2}}{2}\left(1-\frac{f}{g^{2}}\right)
$$

and

$$
\begin{gathered}
f=1-\frac{m^{2} c^{4}}{\gamma_{0}^{4} M^{4} \alpha^{2}} b^{2}+\frac{2 c^{2} K_{B} T}{\alpha \gamma_{0}^{2} V^{2}} \log (g), \\
g=\frac{1}{2}\left[1-\frac{m c^{2}}{2 \gamma_{0}^{2} M^{2} \alpha} b^{2}+\frac{c^{2} K_{B} T}{\alpha \gamma_{0}^{2} V^{2}}\right. \\
+\sqrt{\left.\left(1-\frac{m c^{2}}{2 \gamma_{0}^{2} M^{2} \alpha} b^{2}+\frac{c^{2} K_{B} T}{\alpha \gamma_{0}^{2} V^{2}}\right)^{2}-\frac{4 c^{2} K_{B} T}{\alpha \gamma_{0}^{2} V^{2}}\right]}
\end{gathered}
$$

Equation 34 represents an energy integral for a pseudo particle of unit mass at pseudo time $\zeta$ moving with the pseudo velocity $d b / d \zeta$ with a pseudopotential energy $\psi(b)$. In particular, in absence of the effects of relativistic flow $\left(\gamma_{0} \sim 1\right)$ and thermal pressures of electrons and positrons $(\beta \sim 0)$, the pseudopotential [Eq. [35] ] reduces to

$$
\psi(b)=\frac{M^{2}}{2}\left[1+\frac{4\left(b^{2}-M^{4}\right)}{\left(b^{2}-2 M^{2}\right)^{2}}\right]
$$

which is exactly the same as in Ref. (Verheest and Cattaert, 2004). Introducing the parameter $v_{0}=$ $V / c$ and noting that $\beta \equiv K_{B} T / m c^{2} \ll 1$ defines the regimes of weakly relativistic (classical) plasmas and $\beta \gg 1$ that of ultra-relativistic plasmas, we recast $f$ and $g$ as

$$
\begin{aligned}
f & =1-\frac{b^{2}\left(1-v_{0}^{2}\right)^{2}}{M^{4}[1+\Gamma \beta /(\Gamma-1)]^{2}}+2 S \log g, \\
g & =\frac{1}{2}\left[1-\frac{b^{2}\left(1-v_{0}^{2}\right)}{2 M^{2}[1+\Gamma \beta /(\Gamma-1)]}+S+\right. \\
& \left.\sqrt{\left(1-\frac{b^{2}\left(1-v_{0}^{2}\right)}{2 M^{2}[1+\Gamma \beta /(\Gamma-1)]}+S\right)^{2}-4 S}\right]
\end{aligned}
$$

where $S=\left(1-v_{0}^{2}\right) \beta / v_{0}^{2}[1+\Gamma \beta /(\Gamma-1)]$.

A general discussion of Eq. (34) is almost similar to the Sagdeev's approach for large amplitude 
nonlinear waves. The necessary conditions for the existence of solitary waves are (i) $\psi(b)=0$ and $d \psi / d b=0$ at $b=0$, (ii) $d^{2} \psi / d b^{2}<0$ at $b=0$ (iii) $\psi\left(b_{m} \neq 0\right)=0, \psi(b)<0$ for $0<|b|<\left|b_{m}\right|$ and $\left.(d \psi / d b)\right|_{b=b_{m}} \gtrless 0$ according to when the solitary waves are compressive (with $b>0$ ) or rarefactive (with $b<0$ ). Here, $b_{m}$ corresponds to the amplitude of the solitary waves. It is straightforward to verify that the condition (i) is satisfied. However, the condition (ii) is satisfied for $M>M_{c}$, where $M_{c}$ is the critical value of $M$, given by,

$$
M_{c}=\sqrt{\frac{1-v_{0}^{2}}{1+\Gamma \beta /(\Gamma-1)}} .
$$

Later, we will verify the condition (iii) numerically in two different regimes, i.e., weakly relativistic and ultrarelativic regimes. Furthermore, since the pseudopotential $\psi(b)$ is to be a real valued function, the expression under the square root in $g$ must be either zero or positive, yielding $|b|<\left|b_{m}\right| \leq b_{c}$ where

$$
b_{c}=\sqrt{2} M(1-\sqrt{S}) \sqrt{\frac{1+\Gamma \beta /(\Gamma-1)}{1-v_{0}^{2}}} .
$$

It follows that for some given values of $M, \beta$ and $v_{0}$, the wave amplitude will not exceed the critical value $b_{c}$. The upper limit of the Mach number $M_{u}$ can be obtained in terms of $\beta$ and $v_{0}$ from the condition $\psi\left(b_{c}\right) \geq 0$ as

$$
M_{u}=\frac{\sqrt{2\left(1-v_{0}^{2}\right)}(1-\sqrt{S})}{\sqrt{[1+\Gamma \beta /(\Gamma-1)][1+S(\log S-1)]}} .
$$

Thus, in order that the EP plasmas support large amplitude solitary waves, we must have $M_{c}<M<$ $M_{u}$. In particular, for $\beta \rightarrow 0$ (cold plasmas) and $\gamma_{0} \sim 1$, i.e., $v_{0} \ll 1$ (nonrelativistic plasmas) we have $M_{c} \sim 1$ and $M_{u} \sim \sqrt{2}$, i.e., super-Alfvénic solitons may exist with the Mach number satisfying $1<M<\sqrt{2}$. This is in agreement with the results of Verheest and Cattaert (Verheest and Cattaert, 2004), who reported in nonrelativistic cold electronpositron plasmas. Next, in order that $M_{c}<M_{u}$ holds, the function $A\left(v_{0}, \beta\right)$ must be positive, where

$$
A\left(v_{0}, \beta\right)=2(1-\sqrt{S})^{2}-1-S(\log S-1),
$$

together with $0<S<1$. In what follows, we examine numerically the conditions and different limits of the wave amplitude and the Mach number stated above for the existence of large amplitude EM solitons. We focus our discussion on two particular physical regimes of weakly relativistic $(\beta \ll 1)$ and ultrarelativistic $(\beta \gg 1)$ plasmas. These are demonstrated in the two subsections 3.1 and 3.2 Note that one can, in principle, consider some other finite values of $\beta$, which may be neither much smaller nor much larger than unity, however, a corresponding choice of the polytropic index in between $4 / 3 \leq \Gamma \leq 5 / 3$ may not be appropriate, and can lead to some incorrect results.

\subsection{Weakly relativistic regime $(\beta \ll 1)$}

We consider $\Gamma=5 / 3$. Since $0<S<1$ and $0<\beta \ll 1$, we have two cases of interest (i) $0<v_{0}<\sqrt{2 / 9}, 0<\beta<v_{0}^{2} /\left(1-7 v_{0}^{2} / 2\right)$, i.e., when the upper limits of $\beta$ depend on $v_{0}$ and (ii) $\sqrt{2 / 9} \leq v_{0}<1,0<\beta \ll 1$, i.e., when the upper limit of $\beta$ is independent of $v_{0}$. Figure 1 is the contour plot of $A\left(v_{0}, \beta\right)=0$ showing the possible existence region of solitary waves in the $\left(v_{0}, \beta\right)$-plane. Within the domain $0<v_{0}<\sqrt{2 / 9}$, the ranges of values of $\beta$ change according to case (i). For example, the admissible range of $\beta$ at $v_{0}=0.3$ is $0<\beta<0.13$ and at $v_{0}=0.4$ it is $0<\beta<0.36$. So, smaller the values of $v_{0}$, lower is the upper limit of $\beta$. On the other hand, when $\sqrt{2 / 9} \leq v_{0}<1$ and $\beta$ is independen on $v_{0}$, there is a wide range of values of $\beta$ : $0<\beta \ll 1$ for which the solitary waves exist. However, in all the domains the solitary waves must have a maximum amplitude $b_{c}$, provided the admissible Mach number lies in $M_{c}<M<M_{u}$.

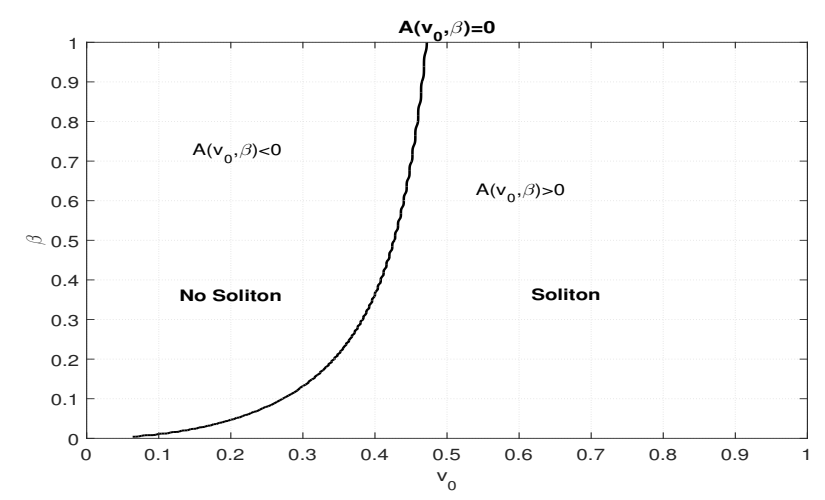

Figure 1: $A\left(v_{0}, \beta\right)=0$ [Eq. [44] is contour plotted to show the existence and non-existence regions of EM solitary waves in weakly relativistic $(\beta \ll 1)$ plasmas.

Figure 2 displays the plots of the lower (solid line) and upper (dashed line) limits of the Mach number within the domain $0 \leq \beta<1$ for different values of $v_{0}$ in two cases discussed before [ $c f$. 
Fig. 11. The subplots (a) and (b) correspond to the case (i) where $\beta$ depends on $v_{0}$, while (c) and (d) that for the case (ii) where $\beta$ does not depend on $v_{0}$. We note that the values of $M_{c}$ are always less than unity, while those of $M_{u}$ can be less than or greater than unity depending on the values of $\beta$ and $v_{0}$ within the regimes. Here, the values of $\beta$ at which both $M_{c}$ and $M_{u}$ coincide are not admissible, because otherwise $M=M_{c}=M_{u}$ would violate the condition for the existence of solitary waves. If we scale $\beta \lesssim 0.05$ to interpret its smallness in the weakly relativistic regime, then from the subplots (a) and (b) of Fig. 2 we find that there are, in fact, two subregimes of $\beta$, namely $0<\beta<\beta_{1}$ and $\beta_{1}<\beta \lesssim 0.05$. In the former regime, we have $1<M_{u}<1.4$, while in the other one has $0<M_{u}<1$. The threshold value $\beta_{1}$ shifts towards lower values as the value of $v_{0}$ is increased within the admissible domain. In fact, for values of $v_{0} \gtrsim 0.7$, the threshold value disappears and only we have $0<M_{u}<1$ in $0<\beta \lesssim 0.05$. Thus, it follows that the EP plasmas with weakly realativistic $(0<\beta \lesssim 0.05)$ energies can support both the sub-Alfvénic $(0<M<1)$ and super-Alfvénic $(1<M<1.4)$ solitons in the regime $0<v_{0}<0.7$, while only the sub-Alfvénic solitons may exist for $0.7 \lesssim v_{0}<1$. From Fig. 2, it is also noticed that the values of both $M_{c}$ and $M_{u}$ decrease with increasing values of $v_{0}$, and they tend to become smaller than unity as $v_{0}$ approaches 1, implying that as the phase velocity of EM solitary waves approaches the speed of light in vacuum, it is more likely that the sub-Alfvénic solitons can exist in relativistic EP-pair plasmas.

In what follows, we numerically examine the variations of the wave amplitude $b_{m}$ [at which $\left.\psi(b)=0\right]$ against the parameter $\beta(0 \leq \beta \ll 1)$ for different values of the Mach number, $M_{c}<M<M_{u}$ and with two different values of $v_{0}$, taking one from each of the regimes $0<v_{0}<\sqrt{2 / 9}$ and $\sqrt{2 / 9}<v_{0}<1$. In these regimes of $M$ and $\beta$, the values of $b_{m}$ are always found to be $\lesssim b_{c}$. We consider (a) $v_{0}=0.3$ when the upper limit of $\beta$ depends on $v_{0}$, i.e., $0<\beta<v_{0}^{2} /\left(1-7 v_{0}^{2} / 2\right)$ and (b) $v_{0}=0.6$ when $\beta$ does not depend on $v_{0}$. The results are shown in Fig. 3. It mainly displays the contour plots of $\psi\left(b_{m} \neq 0\right)=0$ in the $(\beta, b)$-plane. It is interesting to note from subplot (a) that within the domain $0 \lesssim \beta<0.06$ and for a fixed value of $v_{0}=0.3$ in $0<v_{0}<\sqrt{2 / 9}$, the amplitude $b_{m}$ increases with increasing values of $M$ in $M_{c}<M<M_{u}$. However, the same decrease with increasing values of $\beta$

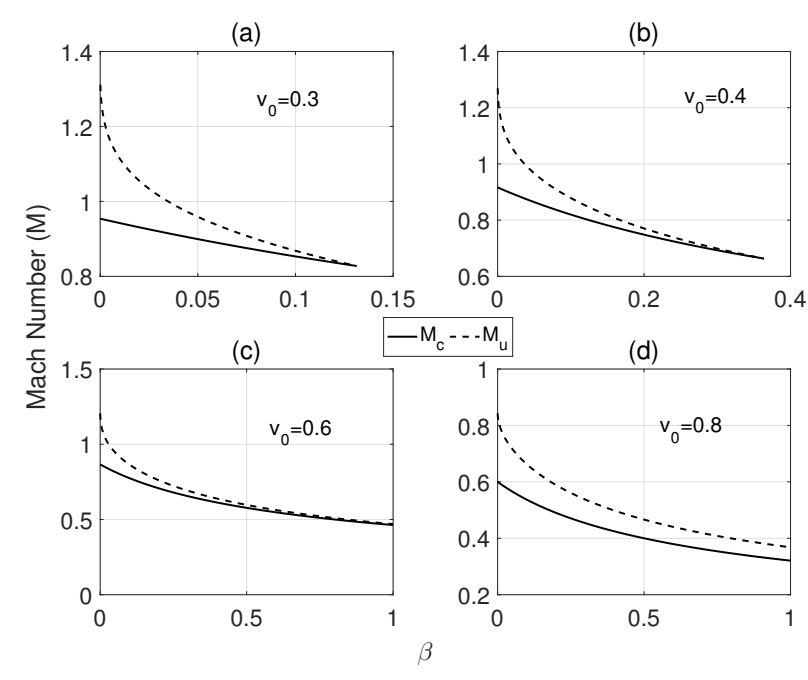

Figure 2: Plots of the lower $\left(M_{c}\right)$ and upper $\left(M_{u}\right)$ limits of the Mach number, given by Eqs. 41 and 43, are shown for different values of $v_{0}$ in weakly relativistic $(0<\beta \ll 1)$ plasmas. The subplots (a) and (b) correspond to the regimes $0<v_{0}<\sqrt{2 / 9}, 0<\beta<v_{0}^{2} /\left(1-7 v_{0}^{2} / 2\right)$, while the subplots (c) and (d) for $\sqrt{2 / 9} \leq v_{0}<1,0<\beta \ll 1$. Note that $M_{c}, M_{u} \leq 1$ for $0.7<v_{0}<1$.

until $1.06 \lesssim M<M_{u}$. However, as $M$ decreases from $M=1.06$ to lower values within the domain $M_{c}<M<1.06$, the values of $b_{m}$ increase in a subinterval $0 \leq \beta \lesssim \beta_{2}$, while those decrease in an other subinterval $\beta_{2}<\beta<0.06$. Here, $\beta_{2}$ is some threshold value of $\beta$ which shifts to higher values as $M$ decreases from 1.06 to $M_{c}$. On the other hand, for a fixed value $v_{0}=0.6$ in $\sqrt{2 / 9}<v_{0}<1$ [subplot (b)], the wave amplitude always increases with increasing values of both $\beta(0 \lesssim \beta<0.05)$ and $M$ $\left(M_{c}<M<M_{u}\right)$. From the subplots (a) and (b) it is also seen that the ranges of values of $\beta$ where $b_{m}$ is defined differ and increase with decreasing values of $M$.

Having obtained various parameter regimes for the existence of EM solitary waves as discussed before, we now plot the profiles of the pseudopotential $\psi(b)$ and the corresponding solitary structures as in Fig. 4 for different values of $v_{0}, \beta$ and the Mach number $M$ in two different regimes (i) $0<v_{0}<\sqrt{2 / 9}, 0<\beta<v_{0}^{2} /\left(1-7 v_{0}^{2} / 2\right)$, $M_{c}<M<M_{u}$ [subplots (a) and (b)] and (ii) $\sqrt{2 / 9} \leq v_{0}<1,0<\beta \ll 1, M_{c}<M<M_{u}$ [subplots (c) and (d)]. As expected, the amplitudes of the solitons exactly correspond to the cut-off values of $\psi$ at $b=b_{m} \neq 0$ (i.e., the points where $\psi$ crosses the $b$-axis). From the profiles of $\psi$ and $b$, the soliton 


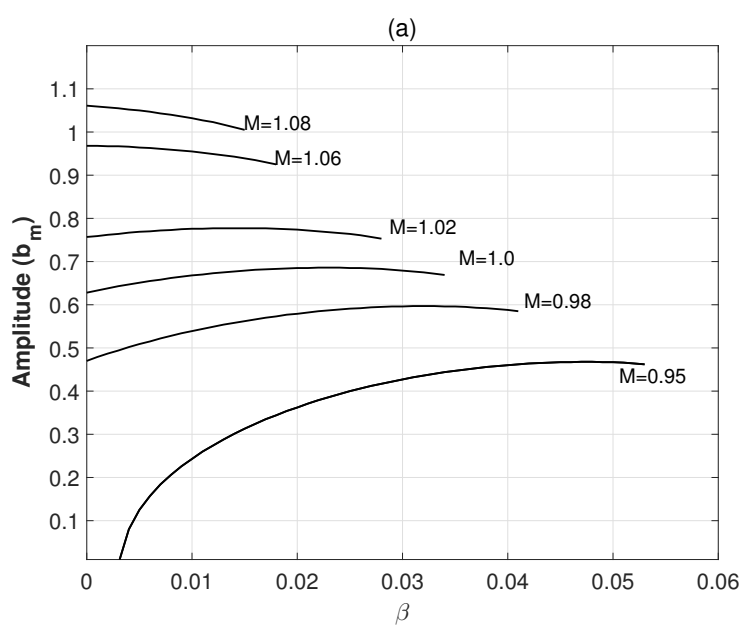

(b)

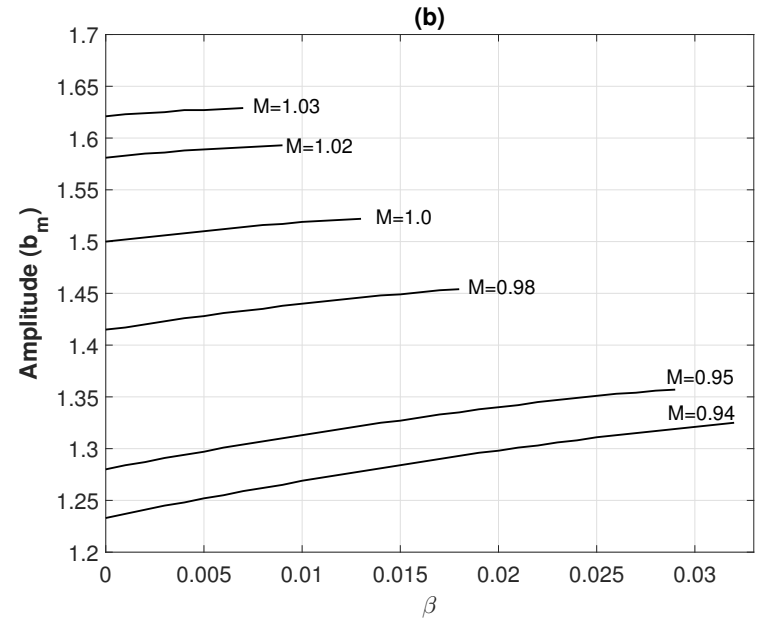

Figure 3: The soliton amplitude $b_{m}$ is shown against $\beta$ with the variations of the Mach number $M$ in two different regimes of $v_{0}$ and $\beta$ : (a) $v_{0}=0.3$ within $0<v_{0}<\sqrt{2 / 9} ; 0<\beta<$ $v_{0}^{2} /\left(1-7 v_{0}^{2} / 2\right)$, and (b) $v_{0}=0.6$ within $\sqrt{2 / 9} \leq v_{0}<1$; $0<\beta \ll 1$. Note that the range of $\beta$ where $b_{m}$ is defined differs and increases with decreasing values of $M$.

widths can also be verified by the formula: width $W=\left|b_{m} / \psi_{\min }\right|$. An enhancement of the amplitude and broadening of the soliton profile (width) are seen to occur with an increase of the Mach number, however, the amplitude increases but the width decreases with increasing value of $v_{0}$ and $\beta$ within the admissible regimes [subplots (a) and (b)]. On the other hand, subplots (c) and (d) show the same qualitative behaviors, i.e., with an increase of any one of $v_{0}, \beta$ and $M$, the amplitude increases and the width decreases.

\subsection{Ultra-relativistic regime $(\beta \gg 1)$}

We consider the polytropic index $\Gamma=4 / 3$. In this case, since $0<S<1$ and $\beta \gg 1$, we can have also two possible regimes similar to the weakly relativistic case, namely (i) $\sqrt{1 / 6}<v_{0}<\sqrt{1 / 5}, 1 \ll \beta<$ $v_{0}^{2} /\left(1-5 v_{0}^{2}\right)$, i.e., when the upper limits of $\beta$ depend on the values of $v_{0}$ and (ii) $\sqrt{1 / 5}<v_{0}<1, \beta \gg 1$, i.e., when the upper limits of $\beta$ do not depend on $v_{0}$. However, looking at the expressions of $M_{c}$ and $M_{u}$, we find that within the regime $\sqrt{1 / 6}<v_{0}<\sqrt{1 / 5}$, the ratio $M_{u} / M_{c}=\sqrt{2}(1-\sqrt{S}) / \sqrt{1+S(\log S-1)}$ varies from 0.9814 to 0.9996 , i.e., $M_{u} / M_{c} \sim 1$ for $\beta \gg 1$. A numerical estimation also reveals that in this regime of $v_{0},|\psi(b)| \lesssim 10^{-9}$ and the soliton amplitude $\left|b_{m}\right| \lesssim 0.01$. So, we are not interested in this short regime of $v_{0}$, and only the regime to be considered for analysis is $\sqrt{1 / 5}<v_{0}<1, \beta \gg 1$.

Figure 5 shows the plots of $M_{c}$ (the lower limit of the Mach number, solid line) and $M_{u}$ (the upper limit of the Mach number, dashed line) within the domain $\sqrt{1 / 5}<v_{0}<1$ for different values of $v_{0}$. We find that both $M_{c}$ and $M_{u}$ decrease with increasing values of $\beta$ and they remain less than unity even for $\beta \gg 1$. Furthermore, it is noticed that the values of both $M_{c}$ and $M_{u}$ decrease with increasing values of $v_{0}$. Thus, it follows that in contrast to the weakly relativistic regime, the EP plasmas with ultrarelativistic energies may support only sub-Alfvénic solitons. Such a feature in relativistic EP plasmas has not been reported before.

Similar to the case of weakly relativistic plasmas, we also show the variation of the soliton amplitude $b_{m}$ for different values of the Mach number $M$ within $M_{c}<M<M_{u}$ and with a fixed value of $v_{0}$ in $\sqrt{1 / 5}<v_{0}<1$ as shown in Fig. 6. It is found that the values of $b_{m}$ increase with increasing values of $\beta$, however, the threshold values of $\beta$ shift to lower ones as the values of $M$ are increased. Since $\beta \gg 1$, relatively lower values of $M$ would favor the existence of EM solitary waves in ultrarelativistic regimes.

The pseudopotential $\psi(b)$ and the corresponding soliton profiles of the magnetic field $b$ are also shown in Fig. 7 for different values of $v_{0}, \beta$ and the Mach number $M$. It is seen that with increasing values of these parameters, the soliton amplitude increases and the width decreases.

\section{Conclusion}

We have studied the nonlinear propagation of purely stationary large amplitude electromagnetic 
(a)

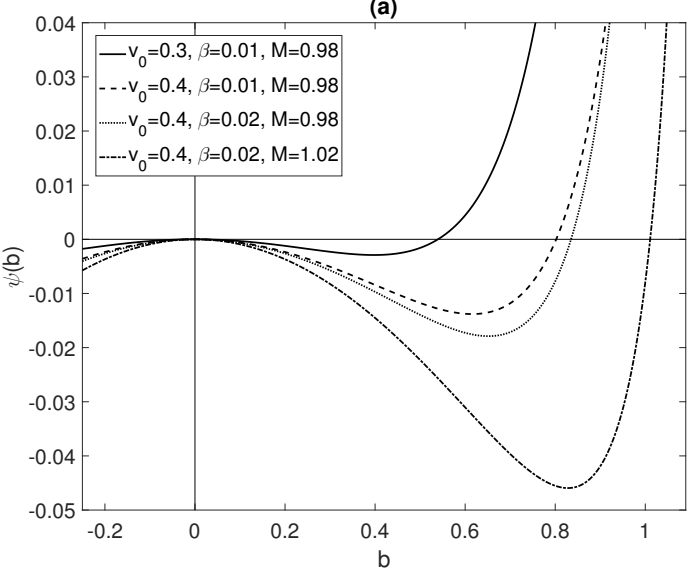

(c)

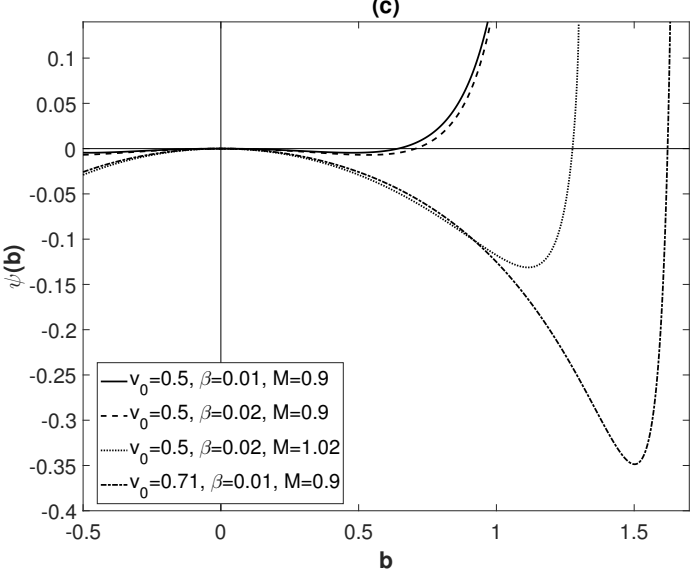

(b)

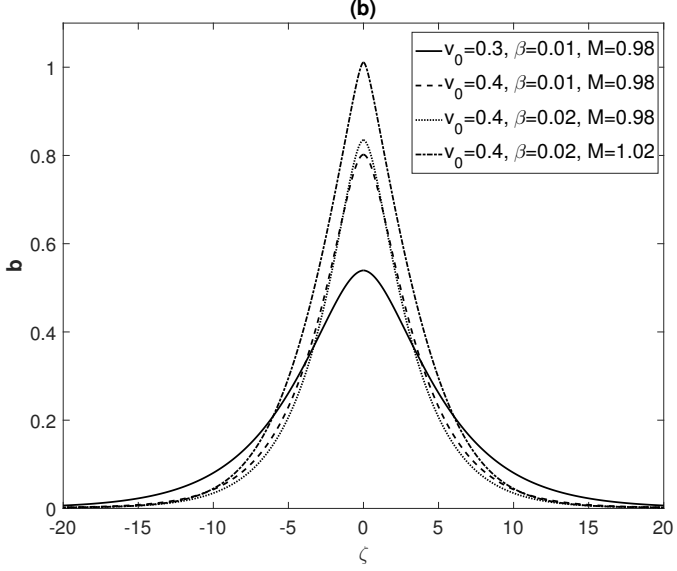

(d)

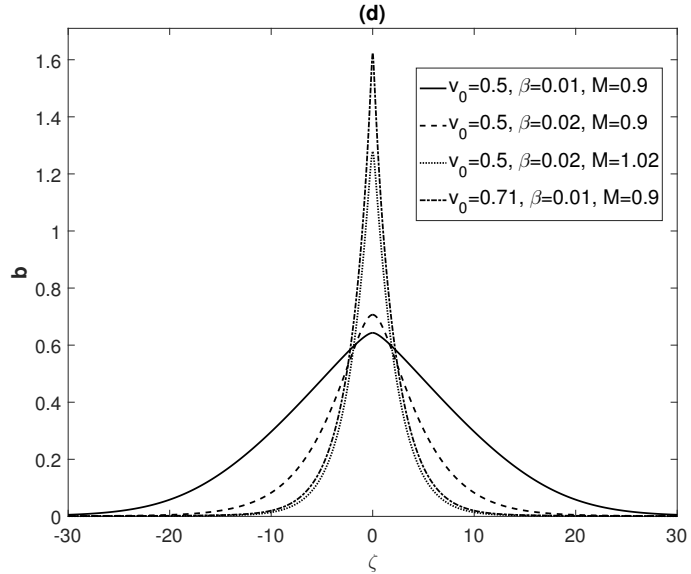

Figure 4: Plots of the pseudopotential $\psi(b)$ [subplots (a) and (c)] and the corresponding soliton profile [(b) and (d)] for different values of $v_{0}, \beta$ and $M$ as in the legends in two different regimes: (i) $0<v_{0}<\sqrt{2 / 9}, 0<\beta<v_{0}^{2} /\left(1-7 v_{0}^{2} / 2\right), M_{c}<M<M_{u}$ [subplots (a) and (b)] and (ii) $\sqrt{2 / 9} \leq v_{0}<1,0<\beta \ll 1, M_{c}<M<M_{u}$ [subplots (c) and (d)].
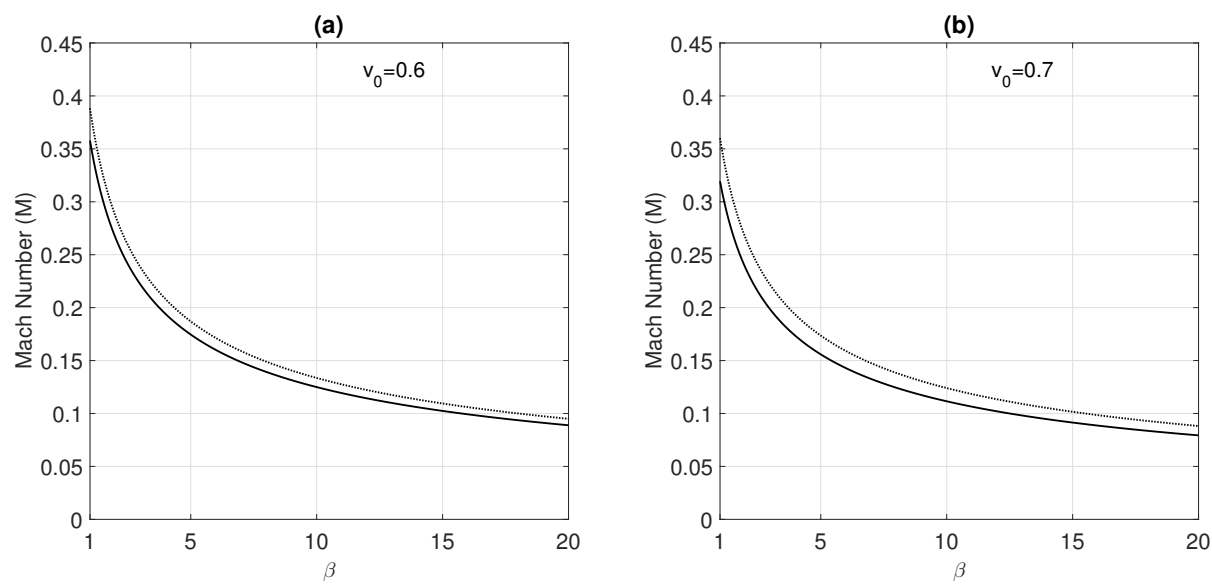

Figure 5: Plots of the lower $\left(M_{c}\right)$ and upper $\left(M_{u}\right)$ limits of the Mach number, given by Eqs. 411) and 43p, are shown for different values of $v_{0}\left(\sqrt{1 / 5}<v_{0}<1\right)$ in ultrarelativistic plasmas $(\beta \gg 1)$ : (a) $v_{0}=0.6$ and (b) $v_{0}=0.7$. 


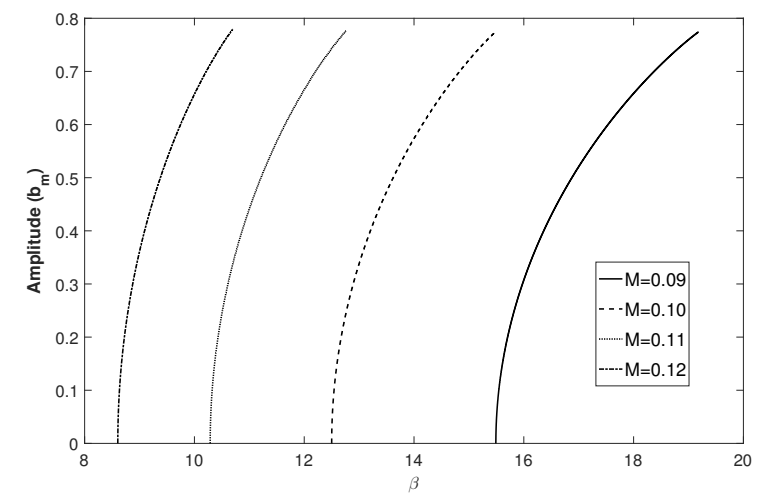

Figure 6: The soliton amplitude $b_{m}$ is shown against $\beta$ in the ultrarealtivistic regime $(\beta \gg 1)$ for different values of the Mach number $M$ and for a fixed value of $v_{0}=0.7$ in $\sqrt{1 / 5} \leq v_{0}<1$.

solitary waves in a magnetized relativistic electronpositron-pair plasma. A fully relativistic two-fluid model is considered which accounts for both the weakly relativistic $(\beta \ll 1)$ and ultrarelativistic $(\beta \gg 1)$ thermal motions of electrons and positrons where $\beta \equiv k_{B} T / m c^{2}$. Thus, previous theory in the literature (Verheest and Cattaert, 2004) is advanced and generalized. Using the McKenzie approach, the system of fluid equations is reduced to an energy-like equation which describes the evolution of EM solitary waves in its own reference frame. Different parameter regimes of the wave phase velocity $v_{0} \equiv V / c$ and the energy ratio $\beta$ for the existence of solitary waves, as well as different limits of the soliton amplitude $\left(b_{m}\right)$ and the Mach number $M \equiv V / V_{A}$ are demonstrated both in the limits of weakly relativistic and ultrarealtivistic energies. It is found that

- In the weakly relativistic limit, EM solitary waves may exist in two different regimes (i) $0<v_{0}<\sqrt{2 / 9}, 0<\beta<v_{0}^{2} /\left(1-7 v_{0}^{2} / 2\right)$ and (ii) $\sqrt{2 / 9} \leq v_{0}<1,0<\beta \ll 1$. The solitary waves can appear as the sub-Alfvénic $(0<M<1)$ or super-Alfvénic $(1<M<\sqrt{2})$ solitons with amplitude $0<b_{m}<2$.

- In the ultrarelativistic limit, EM solitary waves exist in the regime $\sqrt{1 / 5}<v_{0}<1, \beta \gg 1$. In this case, only sub-Alfvénic $(0<M<0.4)$ solitons may exist with amplitude $0<b_{m}<1$.

It is to be noted that both the sub-Alfvénic and super-Alfvénic solitons exist symmetrically for the wave magnetic field $b \equiv B_{y} / B_{0}>0$ or $<0$ owing to the obvious symmetry of EP-pair plasmas with equal mass and opposite charges. This means that the EM solitary waves can propagate as compressive or rarefactive type solitons. The energy integral is expressed in terms of the magnetic field instead of the electrostatic potential as the latter may be relevant for electrostatic solitary waves not for EM waves. Furthermore, we have considered the isothermal pressure law for mathematical simplicity. Instead, one can use the adiabatic pressure law, i.e., $P / P_{0}=\left(n / n_{0}\right)^{\Gamma}$ with polytropic index $\Gamma$, however, in this case, the relativistic fluid equations may not be reducible to the energy integral form (34) either by the McKenzie approach or Sagdeev approach.

To conclude, the nonlinear excitation of EM waves and the formation of solitary structures in pair plasmas are known to have significant relevance not only in space and astrophysical environments but also in laboratory experiments (Sarri et al. 2015). Furthermore, in pulsars and active galactic nuclei with violent surroundings, these nonlinear phenomena would not occur with small amplitude only. In this context, the present theory in magnetized electron-positron plasmas can help understand certain aspects of these stronger nonlinear phenomena with large wave amplitude.

\section{Acknowledgments}

This work was initiated when Sayantan Dutta was pursuing his Master's degree final project in the Department of Mathematics of Visva-Bharati. One of us, GB acknowledges financial support from University Grants Commission (UGC), Govt. of India, under the Dr. D. S. Kothari Post Doctoral Fellowship Scheme with Ref. no. F.42/2006(BSR)/MA/18-19/0096). APM is supported by the Science and Engineering Research Board (SERB), Govt. of India with Sanction order no. CRG/2018/004475 dated 26 March 2019.

\section{References}

Baboolal, S., Bharuthram, R., Hellberg, M.A., 1990. Cutoff conditions and existence domains for large-amplitude ion-acoustic solitons and double layers in fluid plasmas. Journal of Plasma Physics 44, 1-23.

Banerjee, G., Maitra, S., 2015. Pseudopotential approach for dust acoustic solitary waves in dusty plasmas with kappa-distributed ions and electrons and dust grains having power law size distribution. Physics of Plasmas 22, 043708 . 

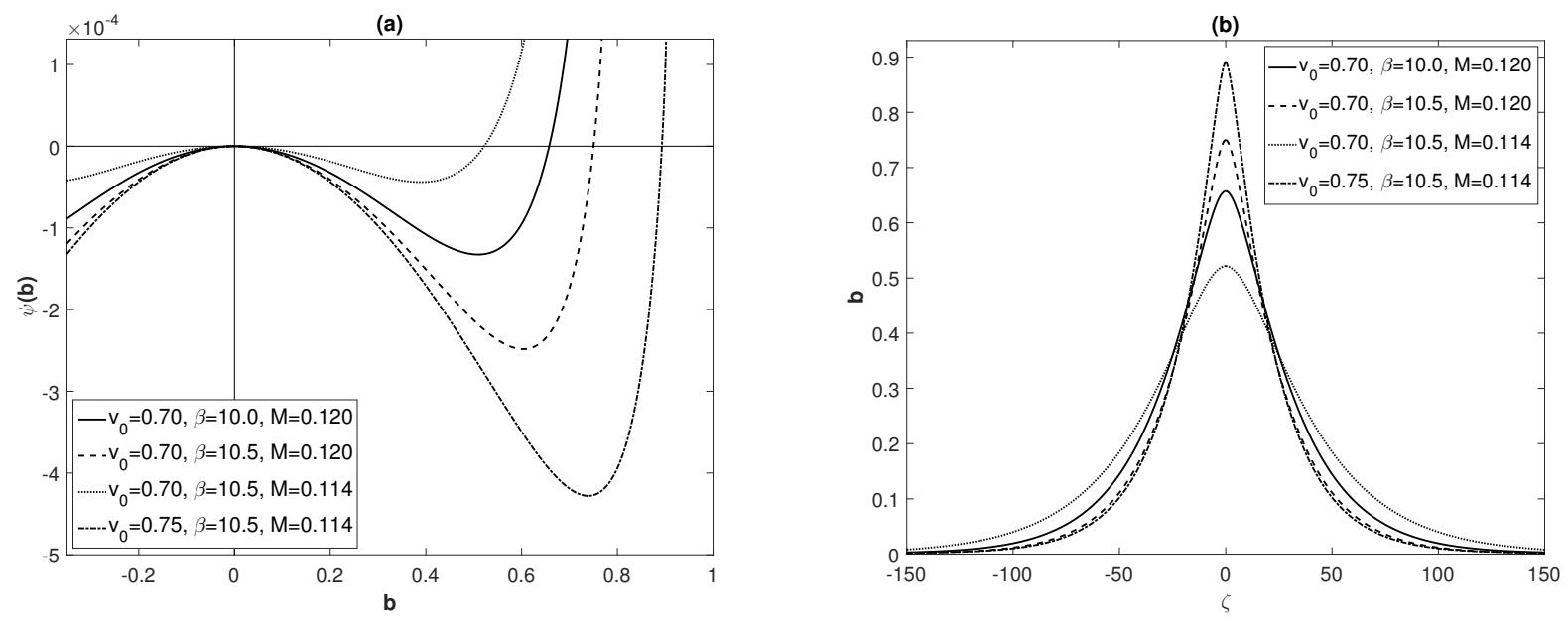

Figure 7: Profiles of the pseudopotential $\psi(b)$ [subplot (a)] and the corresponding soliton [subplot (b)] are shown in ultrarelativistic $(\beta \gg 1)$ regime for different values of $v_{0}, \beta$ and $M$ as in the legends with $\sqrt{1 / 5} \leq v_{0}<1$ and $M_{c}<M<M_{u}$.

Banerjee, G., Maitra, S., 2016. Arbitrary amplitude dust ion acoustic solitons and double layers in the presence of nonthermal positrons and electrons. Physics of Plasmas $23,123701$.

Begelman, M.C., Blandford, R.D., Rees, M.J., 1984. Theory of extragalactic radio sources. Reviews of Modern Physics $56,255$.

Blandford, R.D., Znajek, R.L., 1977. Electromagnetic extraction of energy from kerr black holes. Monthly Notices of the Royal Astronomical Society 179, 433-456.

Burns, M.L., Lovelace, R.V.E., 1982. Theory of electronpositron showers in double radio sources. The Astrophysical Journal 262, 87-99.

Das, A., Bandyopadhyay, A., Das, K.P., 2010. Large amplitude dust acoustic solitary waves and double layers in positively charged warm dusty plasma with nonthermal electrons. Physics of Plasmas 17, 014503.

Gibbons, G.W., Hawking, S.W., Siklos, S., 1983. The very early universe cambridge university press, in: Nuffield Workshop)[21 June 1982].

Goldreich, P., Julian, W.H., 1969. Pulsar electrodynamics. The Astrophysical Journal 157, 869-880.

Gomberoff, L., Galvão, R.M.O., 1997. Modulational instability of a circularly polarized wave in a magnetized electronpositron plasma with relativistic thermal energies. Physical Review E 56, 4574.

Gratton, F.T., Gnavi, G., Galvao, R.M.O., Gomberoff, L., 1997. Self-modulation of a strong electromagnetic wave in a positron-electron plasma induced by relativistic temperatures and phonon damping. Physical Review E 55, 3381

Lightman, A.P., 1982. Relativistic thermal plasmas-pair processes and equilibria. The Astrophysical Journal 253, 842858.

Lightman, A.P., Zdziarski, A.A., 1987. Pair production and compton scattering in compact sources and comparison to observations of active galactic nuclei. The Astrophysical Journal 319, 643-661.

Mace, R.L., Baboolal, S., Bharuthram, R., Hellberg, M.A., 1991. Arbitrary-amplitude electron-acoustic solitons in a two-electron-component plasma. Journal of plasma physics $45,323-338$.

McKenzie, J.F., Doyle, T.B., 2003. A unified view of acoustic-electrostatic solitons in complex plasmas. New Journal of Physics 5, 26.

Miler, H.R., Witta, P.J., 1987. Active galetic nuclei. Springger-Verlag, Berlin , 202.

Misner, W., Thorne, K.S., Wheeler, J.A., 1973. Gravitation. Freeman, San Francisco. p. 763.

Misra, A.P., Adhikary, N.C., 2011. Large amplitude solitary waves in ion-beam plasmas with charged dust impurities. Physics of Plasmas 18, 122112.

Misra, A.P., Adhikary, N.C., 2013. Electrostatic solitary waves in dusty pair-ion plasmas. Physics of Plasmas 20, 102309.

Orosz, J.A., Remillard, R.A., Bailyn, C.D., McClintock, J.E., 1997. An optical precursor to the recent x-ray outburst of the black hole binary gro j1655-40. The Astrophysical Journal Letters 478, L83.

Sagdeev, R.Z., 1966. Cooperative phenomena and shock waves in collisionless plasmas. Reviews of plasma physics 4,23 .

Sagdeev, R.Z., Galeev, A.A., 1969. Nonlinear plasma theory. New York: Benjamin.

Saini, N.S., Danehkar, A., Hellberg, M.A., Kourakis, I., 2011. Large-amplitude electron-acoustic solitons in a dusty plasma with kappa-distributed electrons, in: AIP Conference Proceedings, American Institute of Physics. pp. 357-358.

Sarri, G., Poder, K., Cole, J.M., Schumaker, W., Di Piazza, A., Reville, B., Dzelzainis, T., Doria, D., Gizzi, L.A., Grittani, G., Kar, S., Keitel, C.H., Krushelnick, K., Kuschel, S., Mangles, S.P.D., Najmudin, Z., Shukla, N., Silva, L.O., Symes, D., Thomas, A.G.R., Vargas, M., Vieira, J., Zepf, M., 2015. Generation of neutral and highdensity electron-positron pair plasmas in the laboratory. Nature communications 6, 6747.

Verheest, F., Cattaert, T., 2004. Large amplitude solitary electromagnetic waves in electron-positron plasmas. Physics of Plasmas 11, 3078-3082. 
Verheest, F., Cattaert, T., 2005. Parallel propagating electromagnetic solitons and oscillitons in space plasmas and in relativistic electron-positron plasmas. Physica Scripta 2005,62 .

Verheest, F., Hellberg, M.A., Gray, G.J., Mace, R.L., 1996. Electrostatic solitons in multispecies electron-positron plasmas. Astrophysics and Space Science 239, 125-139.

Wardle, J.F.C., Homan, D.C., Ojha, R., Roberts, D.H., 1998. Electron-positron jets associated with the quasar 3c279. Nature 395, 457-461.

Yu, M.Y., Shukla, P.K., Stenflo, L., 1986. Alfven vortices in a strongly magnetized electron-position plasma. The Astrophysical Journal 309, L63-L65. 